\title{
Functional Bases of Fiber Length and Angulation in Muscle
}

\author{
CARL GANS AND FRITS DE VREE \\ Department of Biology, The University of Michigan, Ann Arbor, Michigan \\ 48109 (C.G.), and Department of Zoology, Universitaire Instelling \\ Antwerpen, Antwerp, Belgium (F.D.V.)
}

\begin{abstract}
The differences in angulation and length observed for the fibers of anatomical muscles may reflect two distinct mechanical requirements: 1) arrangement for pinnation, reflecting an increase in physiological crosssection and 2) arrangement for equivalent placement of sarcomeres, possibly associated with coordination. The observed differences in fiber angulation and length have different effects upon the responses of sarcomeres, specifically on their extent and rate of shortening and on the force they may generate. The basic mechanisms governing these effects and the various arrangements of muscles are reviewed. Fiber length and angulation in the complex M. adductor mandibulae externus 2 of a lizard were measured stereotactically; these values correlate well with the hypothesis that the muscle shows equivalence and demonstrate that angulation for pinnation is less constant. An outline for the study of muscle architecture and function, detailing the kinds of information required to estimate forces and evaluate muscle and fiber placements, is presented.
\end{abstract}

Recently, the functional characteristics of the three levels of muscle architecture have attracted renewed attention. These levels are 1) the organization of the sarcomeres and their placement within fibers, 2) the placement of fibers within muscles, and 3) the location of muscles within organisms. This renewed interest reflects continuing arguments about the relative adaptive value of various muscular arrangements and the extent to which different muscle arrangements represent the phylogenetic history, developmental demands, and results of adaptation to current conditions. Obviously, the physical consequences of distinct architectural arrangements must be understood before one can differentiate among possible alternatives and before one can consider questions of how a structure may be matched to a task. The present study analyzes the theoretical bases of such architectural arrangements.

Striated muscles of vertebrates generally do not insert normally (i.e., at a right angle; Stern, '74); even those muscles crossing only a single joint commonly do not lie parallel to each other. Furthermore, the component fi. bers of individual muscles often differ in length and only rarely are parallel either to each other or to the direction of action of their muscle. Such angulation of muscle and fiber direction is sometimes assumed to be explained entirely by the principles of angulation associated with pinnation (Gans, '82); however, angulation generally is more complex and subsumes a number of supplementary characteristics. Consequently, we need a statement of guiding principles that describes the mechanical implications of muscle locations and allows us to interpret and, ultimately, to test observations.

The principles must permit us to deal with such questions as how much muscle is required to perform a particular task, what is the optimal location of such a muscle mass, how should such a muscle be arranged (in terms of fiber angle, methods of attachment and type of connection), how should the time course of force generation be matched to the functional requirements of the motor system. Answers to these questions become more complex if the musculature performs multiple tasks so that optimization of the totality requires compromises. Most of all, we need to establish the kinds of measurements that are required to answer such questions and obtain information about the degree of uncertainty introduced if any of these measurements are omitted. 
We stumbled onto the need for such an approach during attempts to analyze the muscular apparatus of Trachydosaurus rugosus, a large scincid lizard with a substantial masticatory musculature. Because the species has a simple rotating (single degree of freedom) mandibular articulation (Gans et al., '85), theoretically it should have little need for fine motor coordination. However, Trachydosaurus proved to have a remarkably complex musculature with different muscles interdigitating and with the fibers of individual muscles arranged at angles in multiple planes. This led us to reexamine muscle locations in other species and to consider the possible costs and advantages of the several possible locations of a particular muscle.

The present paper provides a theoretical basis for such comparisons. It reviews the basic arrangements of muscle and then discusses the kinds of angled fibers and their roles. As part of this review, we stress the difference between the angulation of fibers resulting from equivalence and that resulting from pinnation. An appendix includes a data set providing a test of the hypothesis of equivalence. Beyond this, the account provides a discussion of the principles for analyses of architectural systems. We specifically have omitted analyses of the several kinds of curvilinear systems in which muscles enclose fluid-filled spaces, as well as of systems in which force-transmission is related primarily to pressures exerted at right angles to the direction of the fibers (Gans, '82; Gans and Bock, '65). We have included a few general references that discuss muscular organization and that offer more extensive bibliographies (Burke, '78; Gans, '82; Hoyle, '83; Josephson, '75; Loeb and Gans, '86; Mashima, '84; McMahon, '84). However, most of the present analysis is not documented by recourse to authority and should stand or fall by its stated assumptions and internal logic.

\section{SOME PRINCIPLES \\ The contractile system}

The contractile aspect of muscle resides in overlapping thick and thin filaments (Fig. 1). Groups of these filaments constitute myofibrils, which in turn are bound together to form the individual muscle fibers. Each of the two kinds of filaments is interlocked in a pattern that looks like a two-directional trivet, the tines of which slide among those of the adjacent ones. Bridges can form and break among the thick and thin filaments; these bridges tend forcefully to shorten the muscle (McMahon, '84). The thin filaments are spaced by the z-lines, plate-like structures (appearing as lines in lateral view) and

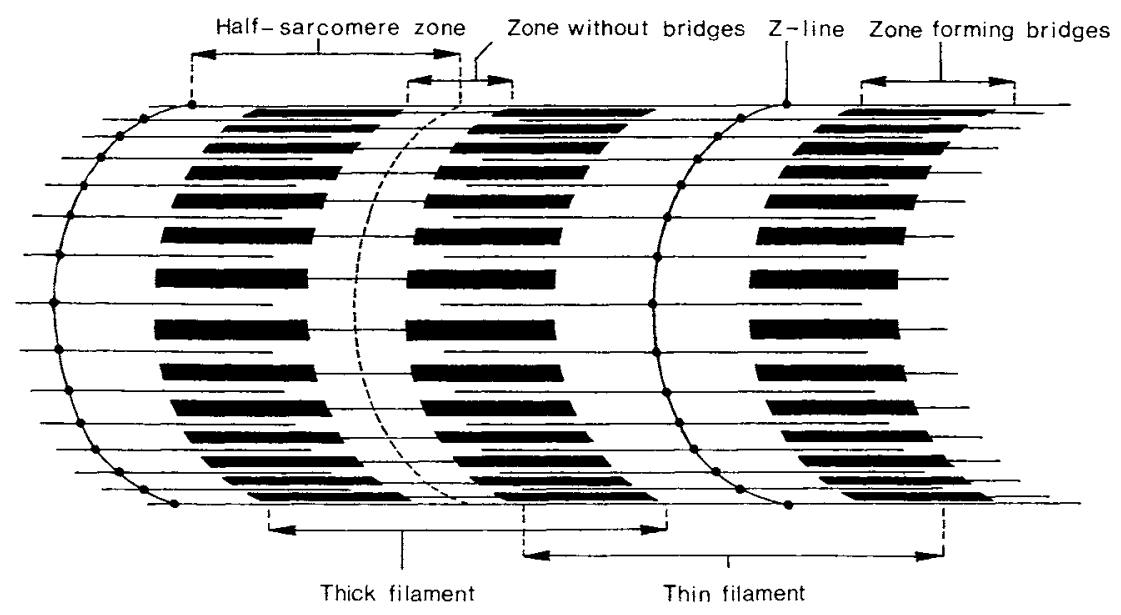

Fig. 1. Diagram of a portion of a muscle fiber showing the thick and thin filaments and the zones on which cross-bridges can form as the trivets slide into one another. The half sarcomeres replicate one another because the two halves of a sarcomere occupy equivalent positions. 
the thick ones by an analogous interconnection near their middle. The degree of overlap of the sliding filaments affects the number of bridges that can form. The unit of contraction, a sarcomere, extends from $\mathrm{z}$-line to $\mathrm{z}$ line including two zones of overlap along the myofibrils. Other considerations being equal, the number of bridges forming among the filaments of a half-sarcomere will thus be proportional to the magnitude of the potential force that the system can generate. (As the bridges in the two halves of a single sarcomere lie in series with each other, the half-sarcomere is actually the unit that determines the contractile action).

Thus, the observed overlap within each half-sarcomere correlates well with the magnitude of the static tension measured for that particular length (Gordon et al., '66). Indeed, the isometric (i.e., no-movement) tension values of the strings of sarcomeres, of the fibers into which they are assembled, and of whole muscle are comparable (Otten, ' 87 ). The isometric force generated by a sarcomere then reflects the number of its parallel filaments and the amount of this overlap at the time of activation.

The length of the zones that may be in contact for any degree of overlap (Fig. 1) is affected, in turn, by the absolute length of the thick and thin filaments and occasionally by their relative numbers (Huddart, '75; Levine et al., '72). The compressive properties of the filaments also affect force production for short sarcomere lengths, as shown along the left side of the length-tension curve (Fig. 2A). As yet, such values have been determined for only a few muscles (Otten, '87).

Force values are calculated and measured for sarcomeres that are stimulated isometrically while they are restrained from movement and fixed at a particular level of overlap. The force produced drops if the stimulated sarcomeres are permitted to shorten (slide into further overlap), and the loss of force reflects the velocity at which shortening (concentric contraction) occurs (Fig. 2B). In contrast, a stimulated fiber will resist extension (eccentric contraction) with more force than it is able to exert in the isometric condition or in active shortening.

\section{Fibrillar arrangement}

Sarcomeres are arranged in series to form single fibrillar strings. All sarcomeres of such a string need to be stimulated approximately simultaneously. Theoretically, the force gen-
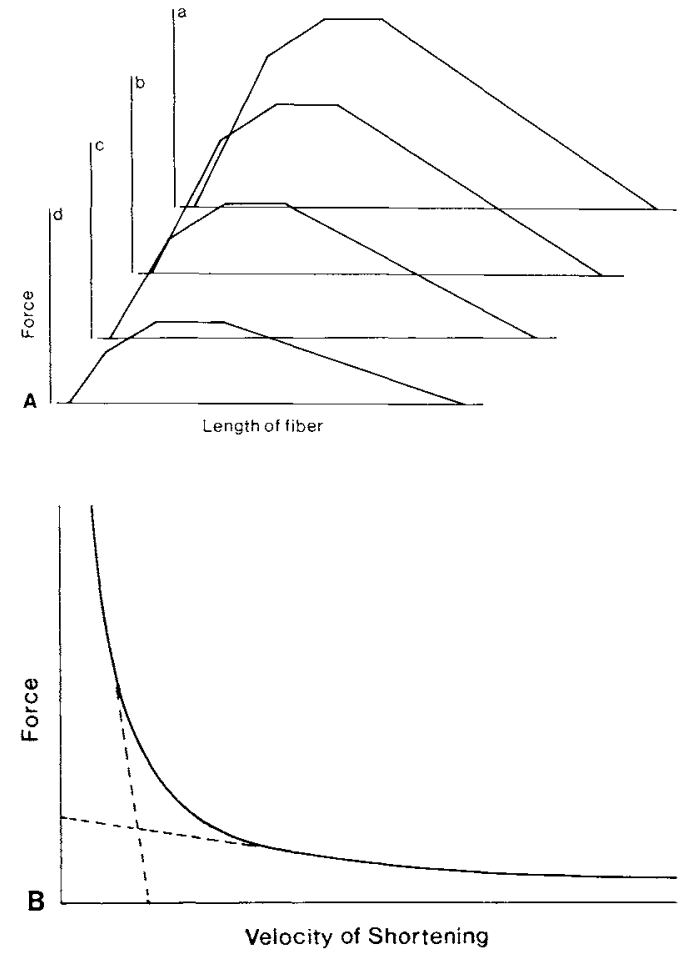

Fig. 2. A. Set of length-tension curves for a single sarcomere showing the isometric value (a) and three different rates of shortening (concentric contraction); velocity for $\mathrm{b}$ is less than for $\mathrm{c}$ and that for $\mathrm{d}$ greater still. The force at lengthening (eccentric contraction) would be greater than that shown in a. B. Sample force-velocity curve to show the general relation. Note that increased velocity produces a disproportionate decrease in the force exerted. Curves are given as indicators of the general force relations and will differ depending upon the muscle tested and the environmental conditions at activation. (A. After Gordon et al., '66, as modified in Gans, ' 82 .)

erated within each string will be independent of the length of the fibril (i.e., of the number of serially arranged sarcomeres comprising it). However, the displacements (and potentially, the velocities) of the ends of the string are additive, rising with the number of sarcomeres included.

In contrast, the arrangement of strings of equivalent sarcomeres in parallel will increase the number of bridges that can form in parallel and, thus, increase the force that the fiber (or muscle) array may exert. (This is the basis of the old concept of physiological cross-section.) Obviously, the shortening of the entire muscle will reflect the summation 
of the unit shortenings of each sarcomere in series, but be independent of the number of strings in parallel.

All other things being equal, the diameter of a muscle fiber will determine the force it can generate, and the length of the fiber (i.e., the number of sarcomeres in series) will determine its relative shortening (see section on Relative and Absolute Shortening).

\section{Activation and contraction}

Muscle fibers are activated by motor neurons. The cell bodies of these neurons lie in the ventral portion of the brainstem and spinal cord. Their axons terminate as endplates on the surface of the muscle fibers. Motor end-plates generally lie near the midlength of vertebrate muscle fibers, but there are exceptions; for instance, the end-plates of frog muscle often lie near the fiber end (Guthe, '81). Each motor neuron innervates a number of muscle fibers, assembling them into a motor unit and serving to coordinate the fibers in parallel. In nondiseased and nonregenerated muscle, the fibers innervated by a motor unit are spaced widely, allowing fibers of other motor units to interdigitate. Because of this interdigitation, the volumes (distance times cross-sectional area) occupied by fibers of motor units also may be either coincident (i.e., centered at the same level of the muscle) or displaced horizontally or longitudinally. Also their centroids may lie at equivalent sites or be displaced.

The activity pattern of the motor neuron determines the contraction pattern of the sarcomeres and, thus, of the fibers; it affects their reaction times and energetic characteristics (i.e., the time required for the fibers to develop tension or to shorten). Thus, the contractile properties of all fibers comprising any motor unit are likely the same. Also, the motor neuron determines the number of times a reaction may proceed and the time until the fiber fatigues. These characteristics are reflected in the enzymatic composition of the bridge-forming proteins and the energetic mechanisms of the myoplasm.

The force and excursion that any sarcomere can potentially produce are a function of the resistance imposed upon the system. Consequently, analysis of muscle function (see below) must consider the inertia and compliance (to applied force) of the elements to which the strings of sarcomeres are attached. This implies that whenever a muscle is involved only in movement or stabilization of elements of the body (for which the inertia is close to fixed), its shortening regime will be different from that of a muscle that produces movement against elements with less predictably varying inertia. Furthermore, the force and excursion characteristics of any muscle also are influenced by the size and number of motor units and their pattern of activation, i.e., if they are activated in series, in parallel, or in opposition to each other.

\section{BASES OF ANGULA'TION Patterns of attachment}

Few vertebrate muscles are oriented such that their stimulation effects pure translation (i.e., absolute rectilinear movement without rotation). Instead, most muscles are oriented to produce rotation of the insertion site and, hence, of the muscle. Many axial muscles of fishes and other ectotherms have their origins and insertions on connective tissue inscriptions. However, cephalic and appendicular muscles tend to insert on rigid skeletal elements that are joined by articulations which limit displacement to particular directions. Rotation of the insertion site (by a change in the joint angle) then modifies either the magnitude or the direction of the vectors introduced by the bridging muscle. In contrast, translational joints facilitate the direct application of forces so that the vector line remains constant throughout movement. In rotating systems, the relative positions of origin and insertion of the muscles reflect a complex relationship between the length of the muscle and the joint angle, as well as between these and the instantaneous moment arm (Stern, '74). This relationship will be trigonometric and curvilinear (Fig. 3).

These considerations indicate that in rotational systems, differently placed muscles will shorten at different rates as the joint angle changes. The force generated by a muscle will reflect the length of its sarcomeres at the instant of activation, as well as the rate at which the muscle is allowed to shorten. Similarly, the time course of force generation is potentially affected by the location of the muscle. The fraction of the origin/insertion distance that is occupied by muscle rather than connective tissue is also of potential importance (as it will affect the relative shortening). Consequently, the moment arm of the sarcomere strings, the excursion likely for the individual sarcomeres, their force-velocity curve, and their length-tension curve interact and must be considered together. 

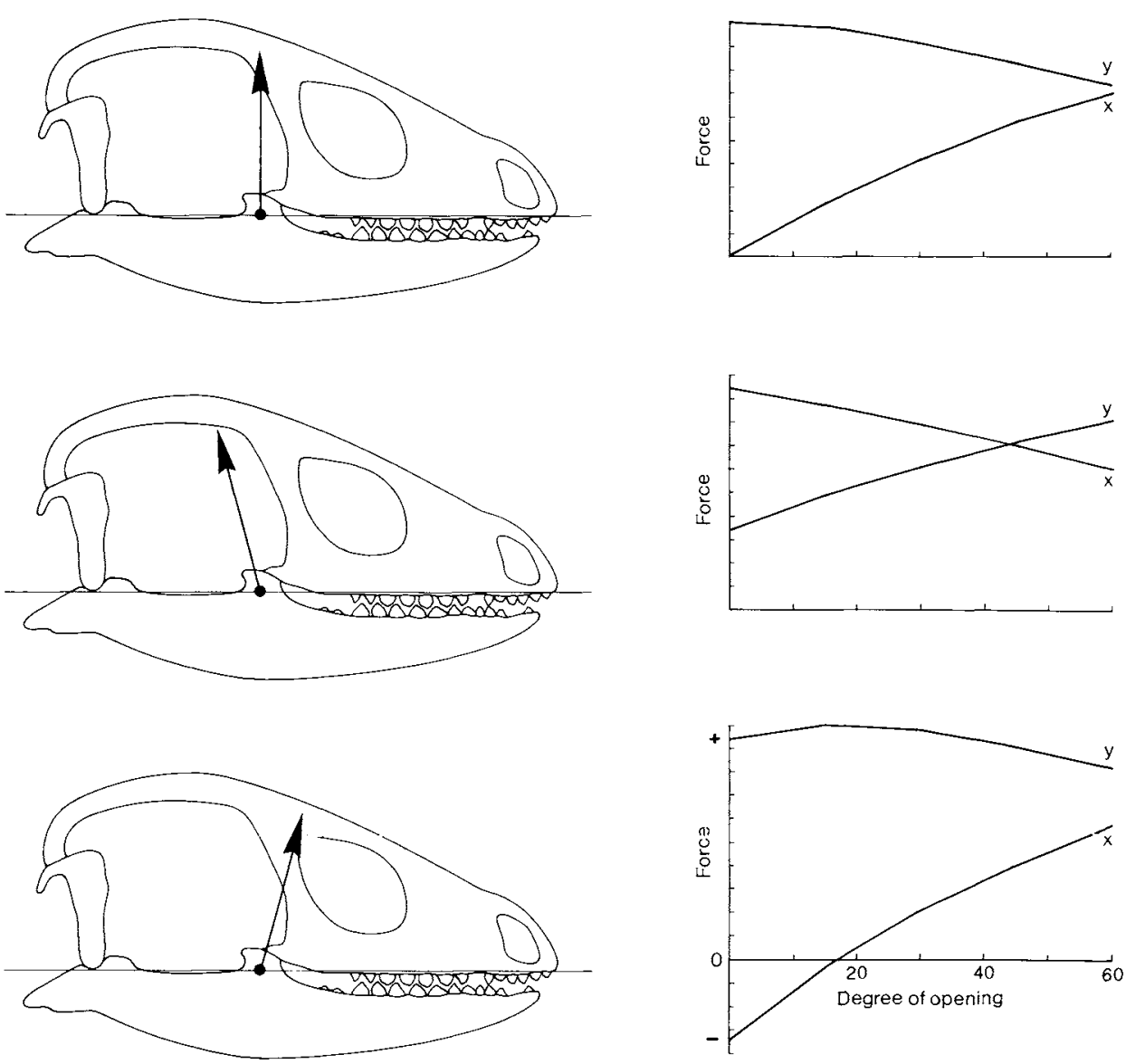

Fig. 3. Diagram showing how the shift of the relative positions of origin and insertion (in this case of a particular mandibular adductor) is reflected in the change of the vector resultants during rotation of the joint bridged by the muscle. Force of contraction is assumed to be equal at each position, so that the length-tension rela-

Such interactions may be complicated even further if muscles are attached across two or more joints, even if the elements thus connected can rotate in synchrony.

The preceding considerations should explain why it is unlikely that selection might act to produce a constant relation of biological function and one isolated variable (e.g., maximum moment arm; Stern, '74, provides some interesting examples). Instead, it seems more probable that selection might have affected the moment directly, i.e., acted to produce a combination of moment arm and generated force (or shortening). The requirements for muscle function may be more complex, e.g., constant moment over a wide range tion must be superimposed to yield real values. $y$ is the resultant at right angles to the moment arm; $x$ is the resultant along the rotating element; +, compressive force toward the joint; -, tension, force away from the joint.

of joint angles, rather than a sharply peaked moment near a restricted portion of the possible range of motion (Gans et al., '85). Also, separate muscles or portions of muscles placed into approximately parallel positions may perform different roles. The kinds of conditions that may be achieved by different combinations of moment arm and shortening are discussed below.

\section{Sarcomeres: placement into functional equivalence}

In any system, coordination among fibers and motor units will be simplest if the sarcomeres are arranged in parallel strings between origin and insertion. All sarcomeres 
in such a parallel array may be assumed to be functionally equivalent; that is, within the limits given below, all sarcomeres can shorten equally and generate equal force for any degree of opening (Fig. 4). In a translational system, the equal-length fibers lying in a rectangular (i.e., straplike) array will be equivalent. In a purely rotational system, involving parallel fibers with parallel moment arms, those fibers lying closer to the joint will have a lower excursion and hence may be shorter. The sarcomeres of both translational and rotational systems will be in physiological equivalence. For rotating systems, this means that equivalence can occur for parallel fibers that lie in a volume that is shaped as a simple, or as a truncated, wedge. The top and bottom surfaces of this wedge represent the surfaces of origin and insertion of the contractile material, respectively, whereas the axis of rotation passes through its apex (Fig. 5). The size of the volume thus circumscribed is clearly limited by the width of the surfaces available for origin and insertion.

The results of the above consideration may appear to be counter-intuitive. Let us assume that the fibers of the above-described muscle

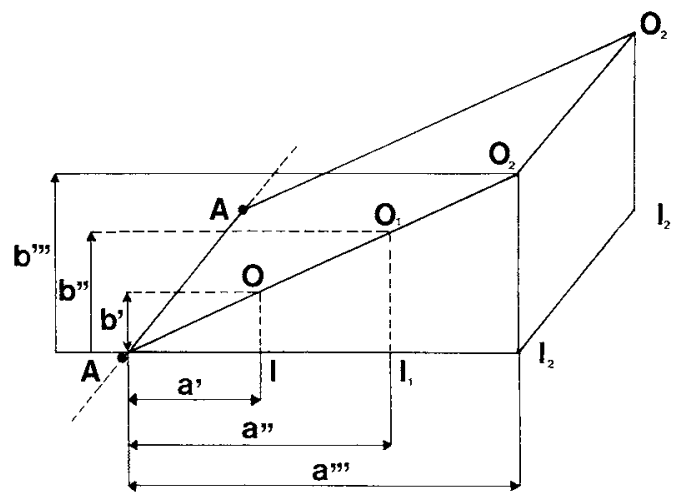

Fig. 4. Wedge of fibers tending to move one surface (AAOO) toward another (AAII) by rotational action about axis A-A. The number of sarcomeres of each fiber, placed in parallel in such a wedge, is likely to be proportional to the length of the fiber ( $I O, \mathrm{I}^{\prime} \mathrm{O}^{\prime}, \mathrm{I}^{\prime \prime} \mathrm{O}^{\prime \prime}$, or $\mathrm{b}^{\prime}, \mathrm{b}^{\prime \prime}, \mathrm{b}^{\prime \prime \prime}$ ) and hence to the moment $\operatorname{arm}\left(\mathrm{a}^{\prime}, \mathrm{a}^{\prime \prime}, \mathrm{a}^{\prime \prime \prime}\right)$. Each sarcomere, in such a wedge of fibers placed in parallel, will produce an equal force and is likely to produce equal moment for equivalent shortening and to induce equal shortening for equivalent moment. This falsifies the common assumption that it is more advantageous to place a muscle (of specific mass) more distally from a joint capable of a set angular excursion.

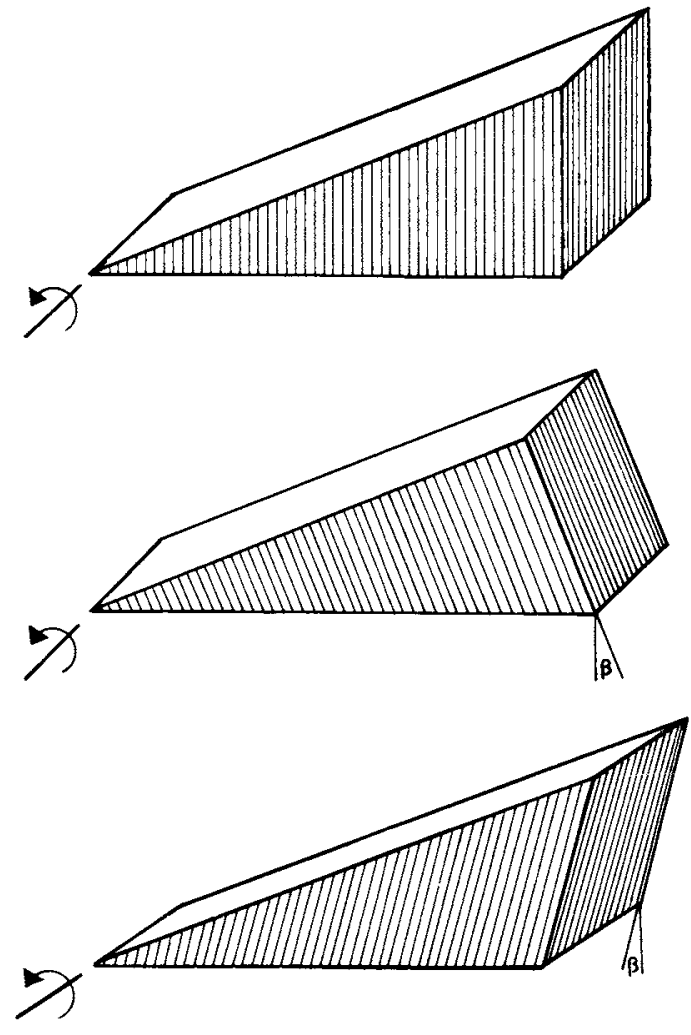

Fig. 5. Diagram illustrating several simple wedges containing fibers with functionally equivalent sarcomeres. Note that the moment arms of the fibers within each wedge are parallel so that each has equal angles of equivalence $(\beta)$.

lie in parallel. Each sarcomere in the (parallel-fibered) space then tends to achieve equivalent absolute shortening and force generation, no matter what the rotation; it is easy to show that each such sarcomere will contribute equally to the moment generated in the moving element. Hence, muscle mass rather than muscle placement determines the moment generated by the array. Strings of sarcomeres positioned further from the center of rotation will have longer moment arms; however, such strings also must be longer, because the requirement for absolute shortening (excursion) of the muscle will increase in proportion to the length of the moment arm. Hence, because equivalent shortening simply requires that a greater number of sarcomeres be placed in series, the contribution of each sarcomere remains constant (cf. Fig. 4). 
Within the overall wedge-shaped space (defined by the surfaces of origin and insertion), one may define truncated wedges, each having equivalent volume. Such wedges of equivalent mass should produce equivalent moment, displacement, and velocity. Superficially, all of these wedges might be assumed to be functionally identical; however, their rotational inertia will increase as the square of the distance between the center of mass and the center of rotation. This differential inertia of the distal element will affect the dynamics of the rotating limb.

The wedge-shaped space, thus defined, represents the maximum volume into which sarcomeres driving a rotating system can be placed in functional and geometric parallel. However, sets of sarcomeres can be placed into purely functional (but not geometric) parallel by arranging them at equivalent angles to their sites of insertion (i.e., by maintaining each fiber at an equivalent angle to the moment arm; see below). This design increases the space available for the placement of muscle fibers beyond that provided by the volume of a simple wedge (Fig. 6). The design requires use of tendons and aponeuroses to increase the surface areas available for the attachment of muscle fibers. However, equiv. alent shortening and force production by the sarcomeres (to provide equivalent rotation of insertion sites) will be achieved only under specific conditions. These conditions are that 1) fibrils are maintained at equal angles relative to lines drawn from their insertion site to the center of rotation and 2) that the length of each fibril is proportional to the length of its moment arm (cf. Fig. 5 for both). The latter demand appears to explain the general angulation of fibers (in the plane of rotation) within certain muscles (Fig. 7; Gans et al., '85). Utilization of multiple wedge-shaped spaces generates a complex architecture of the muscle insertion sites that leads to the use of aponeuroses with which the fibers must be angled to maintain equivalence. It also demands the secondary angulation (discussed below under the heading of pinnation) which occurs at right angles to the plane in which rotation proceeds.

\section{Sarcomere equivalence}

Part of the following discussion is based upon the explicit assumption that simultaneously activated sarcomeres of multiple motor units show some kind of equivalence. This assumption of equivalence is based, in turn, on three other markedly different and mutually independent operational assumptions. 1) All of the sarcomeres in a muscle fiber should contract equally. However, the sarcomeres lying closest to the motor end-plate will be activated prior to those further away and should encounter less initial resistance. 2) All of the fibers of each motor unit should show equivalent numbers of sarcomeres (relative to their moment arm), if only because the fibers are likely to show an equivalent developmental (growth-associated) response involved in determining the number of sarcomeres that will form in series. 3) All of the multiple motor units of potentially equal action and activation within a large muscle, should show sarcomere equivalence; this derives from the consideration that the inter-

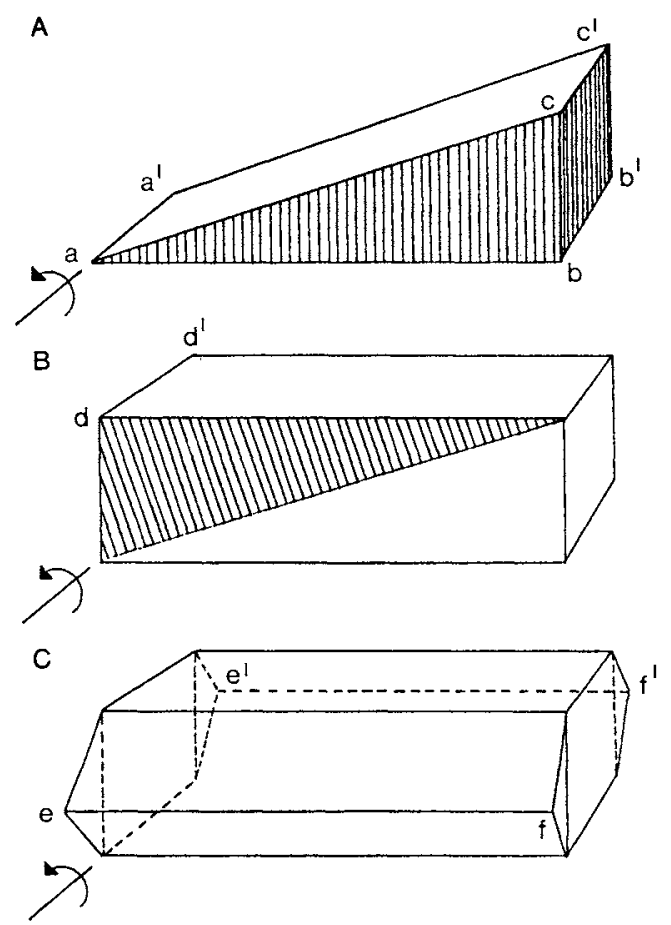

Fig. 6. Ways in which the volume of an equivalently placed muscle may be modified to exceed the fundamentally wedge-shaped pattern (A). Note that in order to maintain equivalence, the supplementary volumes must in each case be filled with fibers lying at angles differing from those in the initial wedge and influenced by the way tendons and aponeuroses may be placed. Generally, packing demands will require that the muscle be pinnate (cf. Fig. 9). Each modification (top to bottom) in creases the total volume of muscle (After Gans et al,, '85). 


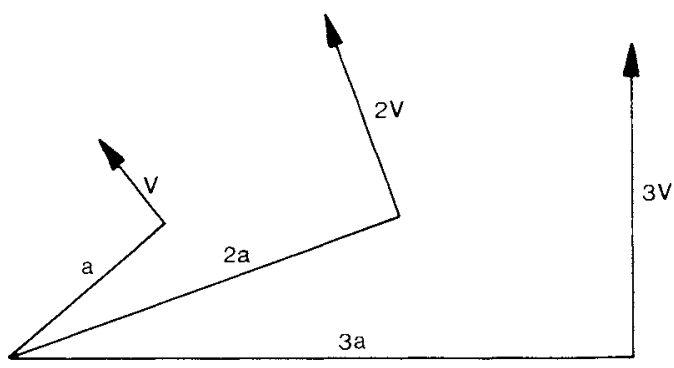

Fig. 7. Diagram illustrating that complex muscles can remain functionally equivalent if the length of each fiber $(\mathrm{V}, 2 \mathrm{~V}, 3 \mathrm{~V})$, equivalent to its number of sarcomeres, is proportional to the length of its moment arm (a, 2a,3a).

neurons activating groups of such motor neurons can be expected to match their physiological and morphological pattern to that of muscle spindles controlling particular movements. However, if particular subsets of motor units have multiple functions, equivalence need not be expected.

There are three additional corollaries for sarcomere equivalence: 1) The absolute length of each sarcomere is equivalent; thus, all thick and thin (respectively) component filaments have the same length. Similarly, the chemical structures of the filaments, of the mitochondria, and of the myoplasm are equivalent. 2) The absolute range of shortening (i.e., the portion of the length-tension curve across which shortening proceeds) is equivalent for each set of sarcomeres. 3) All activated sarcomeres of a given muscle shorten equivalently for a given excursion of the joint.

Each of the latter three assumptions for sarcomere equivalence may be falsified under different conditions and with distinct implications, as follows:

1. The properties of muscle fibers differ depending upon fiber type. Thus, there may be variation in time course of force generation, fatigability, and similar characteristics. Moreover, the several chemical, physiological and histochemical systems proposed differ among organisms (Focant and Pequeux, '85; Focant et al., '85; Vandewalle et al., '83). In addition, the lengths of the thick and thin filaments have recently been shown to differ among muscles within some animals (Akster, '84).

2. There is evidence suggesting that fiber length may differ substantially within some muscles. For instance, the nearly vertically positioned fibers near the point of origin of the frog gastrocnemius often have been reported to be shorter than the laterally placed (and clearly pinnate) fibers. In this case, the concept of equivalence cannot be applied, because several portions of a muscle show different excursions and contribute differently to the overall action produced by the muscle.

3 . The further a point on a moving element lies from the center of rotation, the greater its linear excursion for a particular angular excursion. Hence, muscles that produce rotation and have wide fleshy insertions impose potentially different regimes of absolute shortening upon the parallel strings of sarcomeres. For such muscles, the physiological equivalence of sarcomeres may be potentially different from the morphological equivalence of the muscle fibers.

This physiological equivalence, which pertains only to individual muscles has been tested, but only rarely, although we have evidence that the sarcomeres of various muscles attaching to the mandibles shift through different ranges during mandibular movements (Herring et al., '84; Weijs and van der Wielen-Drent, '82, '83). It would be interesting to determine whether the fibers of a muscle each have sarcomeres with distinct lengths, and whether muscle fibers that are activated simultaneously show sarcomere equivalence.

The best evidence we have for this system considers fiber length within a muscle, rather than sarcomere number. Measurements of the fiber lengths of complex muscles analyzed for this are in accord with the prediction that they relate to the length of the moment arm (cf. Gans et al., '85; Appendix) and by implication fits the prediction of sarcomere equivalence. For these reasons, the models that follow accept the reality of sarcomere equivalence. However, the hypothesis needs further testing. Hence, the analysis outlined in the last section of this paper contains the proposal that there be a test for fiber length and, by implication, for sarcomere heterogeneity. Should this be discovered, the models that follow will be easily modified.

\section{Tendons and aponeuroses}

The shift of the sites of muscle attachment from simple planar surfaces to more complex ones (Fig. 6) requires the use of aponeuroses and tendons for insertion of muscle fibers. These soft tissues generally are compliant, 
primarily resisting tensile force and bending markedly whenever loaded in compression. Thus, not only the change of angle between origin and insertion of the contracting muscle fiber is important, but also the potential displacement of the connective tissues. In this system, the positions of soft tissue attachment to the underlying bony base do not remain stable, and so the requirement for equivalent shortening of the distance between origin and insertion is not met.

Elastic extension of connective tissues can be reduced by thickening the tendons. A fixed attachment zone of soft tissues can be achieved either by stiffening the connective tissues (perhaps by ossification) or by constraining them or the attached muscles among bony tissues that resist inappropriate deformation. Occasionally, a stiffening effect is obtained by tensing the connective tissue through action of certain muscle fibers that are inserted at angles to the main line of force (Gans et al., '85). The angles at which such stiffening fibers are located obviously will differ from the fiber placements required to achieve functional equivalence.

Contracting muscle fibers and their connective tissue attachments will tend to conform to straight lines even in rotating systems. Whenever sets of fibers attach to a single sheet, one can see either one or a combination of two potentially alternate arrangements. In the first, the individual muscle fibers rotate separately and independently. In the second arrangement, rotational capacity is restricted to the entire muscle mass, which shifts about its attachments to bone. After rotation of the muscle, the muscle fibers occupy a straight line between the sites of origin and insertion for the entire muscle.

\section{Performance line}

For any simple rotating system, comparison is facilitated by drawing a "performance line" from the center of rotation to the site at which the element exerts a moment onto external objects (Fig. 8). The length and position of the performance line are necessary to understand the magnitude of the bending moments that must be supported by the skeletal material. This line also is of interest for a simple system of equivalent performance, because the moment arm of muscle fibers will shift as the insertion site departs from this line. The ratio between the normal distance from insertion to the performance line

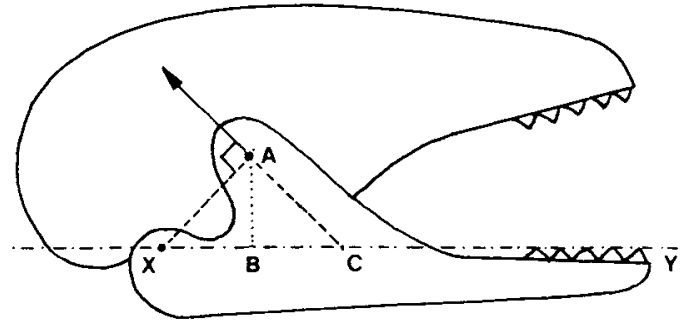

Fig. 8. Sketch showing the moment arm A-X and crossing site (C) relative to the performance line $\mathrm{X}-\mathrm{Y}$, shown for the example of an adductor muscle on a mandible. $A-B$ is the normal distance from insertion site to performance line. If the ratio $A-X / A-B$ is constant within such a muscle, its sarcomeres will lie in functional equivalence.

and the length of the moment arm will reflect the angle of equivalence (cf. Fig. 7).

The performance line (in terms of this ratio) then provides a simple estimate of the homogeneity of fiber angles within a muscle. The simplest ratio compares 1 ) the distance from the joint to intersection of the musclefiber force vector with the performance line (X-C, Fig. 8) and 2) the muscle fiber moment arm (A-B, Fig. 8). If all of the fibers of a muscle have similar ratios, they will show minimal differences in attachment angles. If the ratios for the insertion of the individual fibers differ within a muscle, so will their intramuscular angulation. The relationship is trigonometric, and the effect of deviation differs with the magnitude of the ratio; deviation is greatest for ratios near 1 and becomes insignificant below 0.2 and above 4 . In the latter range, the variation of fiber angles is often likely to be less than the error of measurement. The fibers with such ratios may be assumed to act as if they effect simple translational movements.

The above test will be more complex whenever the lever system induces complex movements; for instance, if the line on which the tooth cusps lie does not pass through the instant center of rotation (Gans, '74, for examples). Then it may become necessary to establish several performance lines and perhaps to check whether different subsets of motor units are active for each. Regional nonequivalence of fiber angle might be an indicator of such subdivision.

\section{Pinnation}

Thus far, muscle fibers have been considered as if they were attached to the edges of 
tendons and aponeuroses so that their vectors were linearly continuous with the collagen fibers. However, muscle fibers rarely attach only in this manner. Instead, surfaces of attachment generally are increased by allowing muscle fibers to connect to the sides of tendons and aponeuroses. The angles of attachment to tendinous surfaces differ from those considered above; they are the classical angles of pinnation (Gans, '82; Gans and Bock, '65). In a strap muscle, pinnation occurs in a single plane (which may twist along the length of the muscle). In a wedge-shaped muscle, this classical form of pinnation usually occurs in planes at right angles to those of the fiber angulation established by the above-described demand for functional equivalence. Because the two effects are independent, the fibers of a particular muscle tend variably to respond to both of them.

Unlike fiber angulation designed to emphasize functional equivalence, pinnation in the classic sense involves a cost. The force vectors generated by a pinnately arranged fiber can be resolved into two components-one in the direction of movement and transmitted via the tendon of insertion, the second a lateral component that does not contribute to movement. This lateral component can be balanced by push of the soft tissues against mechanical stops or by the action of opposing, pinnate (bipinnate) fibers that insert on the opposite sides of the aponeurosis.

In regularly pinnate arrays, all fibers of a fascicle are of equal length, and those fibers on one side of the aponeurosis lie parallel to one another. Often the aponeuroses of origin and of insertion retain a parallel position and equivalent distance to each other during contraction. The mass of the muscle then shifts toward the fixed site along the line of action, so that the muscle does not swell. However, this functional design does not apply to pinnate arrays that are framed entirely in soft tissues, because the force-couples incurred within any unipinnate portion of such arrays will induce local rotations and deformations of the sets of fibers (Gans, '82).

Pinnation has several advantages which compensate for the loss of useful force with angulation. The most obvious is that the distance between the origins and insertions of individual fibers (i.e., their lengths) can be less than that of the entire muscle. If it is assumed that shorter fiber length is physiologically desirable, then the corollary follows that pinnation greatly increases the possible types of fiber arrangements for any particular muscle mass. Thus, the shape of the space occupied by the muscle can be modified without affecting the force transmitted by the tendon. In short, pinnation facilitates a variety of packing arrangements for muscle fibers in the space available without the need for modification of fiber length. Sometimes the ability of pinnate muscles to shorten without swelling of the muscle mid-region is advantageous (Gans and Bock, '65).

These characteristics of pinnation generally have been referred to for muscles transmitting translational forces at their tendons or for those in which rotation in the muscle is so small that it could be ignored. For muscles arranged to effect rotation under conditions of functional equivalence for the sarcomeres (i.e., for muscles that are placed so as to produce vectors with directions that change significantly as they contract), the angulation of fibers resulting from pinnation must be determined in a plane at right angles to the axis of rotation. Furthermore, the effect of this angulation is independent from that resulting from equivalence. Hence, the angle of pinnation should not be measured relative to some abstract axis (e.g., that of the head), but should be measured relative to the plane of the aponeurosis and the axes of movement. If the movement involves rotation about more than a single axis or a combination of rotation and translation, the analysis may have to proceed in terms of instant centers of rotation of the moving elements. These two distinct influences produce the architecture of mastication systems, which is complex in three dimensions (Fig. 9).

\section{Angulation and lateral translation}

Angulation of fibers within a muscle either is a design constraint of meeting the functional equivalence of sarcomeres within a muscle or conditions of classic pinnation. However, some patterns of angulation of fascicles or entire muscles reflect distinct tasks, such as purely translational shifts of the insertion site. This translation can be effected by differential activation among individual fibers (motor units) of a single, radially arranged muscle (Gaunt and Gans, '69), among slips of a muscle (such as the serratus of cats), or among several muscles. The movement produced can be uniaxial (1 degree of free- 


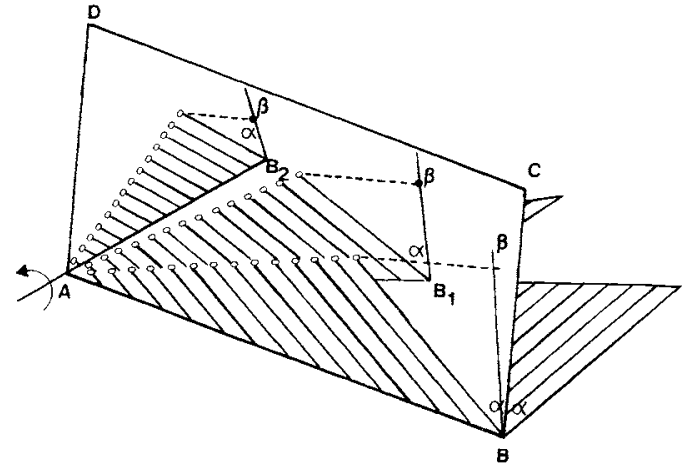

Fig. 9. A complex muscle can combine pinnation and functionally equivalent placement of sarcomeres. The surface A-B-C-D represents an aponeurosis of a bipinnate muscle rotating about an axis passing normally through it at $\mathrm{A}$. The sets of parallel lines represent muscle fibers originating at the small circles and inserting on the aponeurosis. The angle $(\alpha)$ is the angle of pinnation; it is illustrated as being equivalent through the array. The angle $(\beta)$, lying in the plane of the aponeurosis, is the angle of equivalence (cf. Fig. 5). It depends upon the ratio between moment arm (A-B) and the site at which the vector line crosses the performance line. The solid lines join the insertions of fibers that have parallel moment arms.

dom in translation), or have up to 3 degrees of freedom. Consequently, the placements of muscle fibers can be simply linear in opposed directions, planar (tending to shift the insertion site along a surface), or three-dimensional (perhaps radiating outward from an insertion site). The need for translation can lead to angulations among sets of fibers that lie along a plane surface, around the surface of a cone, or throughout the volume of the cone.

The closer the origin or insertion of a normally (i.e., at $90^{\circ}$ ) inserting muscle fiber to the center of rotation (joint), the less the absolute fiber shortening required to rotate the element through a specified angle. As the angle of insertion of the muscle fiber on the performance line shifts from the perpendicular, there is a reduction in the absolute shortening required to effect a particular angular rotation. Of course, location of either origin or insertion close to the center of rotation will produce an equivalent response, except that shallow insertion along the rotating portion of the linkage increases the rotational inertia.
Muscles that insert close to normal (i.e., at right angles) on a performance line and near the center of rotation of the distal element mainly generate vector resultants that enhance rotation of the element. Consequently, only a minor resultant of the total force tends to act along the performance line. As the insertion site shifts outward (i.e., further away from the center of rotation) along the performance line, this component, which is "ineffective" for rotation of the element, increases. The component can compress or unload the portion of the rotating element that lies between the insertion site and the center of rotation, and if the joint permits translational shifts, the insertion site can shift relative to the center of rotation. This is of benefit in propalineal jaw movements in which much of the food reduction reflects horizontal (transverse and anterior-posterior) translation of the mandibular dental batteries relative to the maxillary ones.

Forces that act along the performance line may present problems at the joint. The rotating joints of vertebrates differ from those designed by industry, because in organisms, these joints tend to be constructed as more or less shallow cup supports (and tend to permit rotation through an angle of less than $360^{\circ}$ ). In some cases, vertebrate joints facilitate both rotation and translation, for instance, by the intercalation of meniscal discs. Horizontal forces acting along the bone (the rotationally "ineffective" resultant) can effect this translation. However, translational movements often are restricted to part of the combined rotation/translation cycle. Also, the resultants of muscle force, both in the direction of rotation and of translation, will change as rotation proceeds (cf. Fig. 3). The tendency of a resultant to effect disarticulation at these joints may be counterbalanced by opposing and approximately equal resultants of forces imposed by other muscles. The potentially dislocating forces also can be reduced by functional subdivision of muscles and temporally discrete activation.

Some authors have suggested that some muscles form slings that tend to balance each other, so that the forces potentially acting across joints (for instance, across the mandibular condyle) can be assumed to drop to zero. There seems to be no factual basis for this idealistic assumption. Analysis of any system must consider, at least, the extremes of movement, because the resultants of the vec- 
tors generated by the several muscles can undergo profound shifts throughout rotation (cf. Fig. 3).

\section{Relative and absolute excursion}

The ends of a stimulated muscle incur an absolute excursion and exert an absolute force at an absolute velocity. However, these absolute values generally are determined by the resistance and velocity imposed by the external elements to which the muscle is attached. This is the real basis for the concept of origin and insertion, because the attachment that encounters the most resistance moves the least. Understanding the relationship between the absolute values of excursion and the relative values incurred by each sarcomere is critical, because it is the movement at the sarcomere site that determines the load that can be generated.

Simultaneous stimulation of the fibers of a motor unit need not lead to their simultaneous or equivalent shortening. The shortening stimulus passes outward from the site of the motor end plate at a finite velocity that is dependent on the type and the diameter of the fiber. However, muscle fibers can be constrained irregularly by the connective tissues attaching between them, producing local irregularities in the way they are loaded. Furthermore, the angles of the action lines of the fibers within a motor unit and their rate of shortening can change differentially during rotation of the joint.

Within the above limitations, simple physical considerations indicate that the static tensile force across a parallel-fibered muscle will be equal at any cross-sectional level between origin and insertion. In short, each sarcomere of a string of serially arranged sarcomeres potentially will generate (or have to resist) equal force. Whenever strings are placed parallel with each other, the forces generated by each will be additive; hence, the force of the entire muscle will be the sum of that of its parallel strings.

However, the excursion relations are different. The absolute excursion of the end of the entire muscle will be equal to the absolute excursions of each of the parallel strings of sarcomeres. Also, the absolute excursion of each string will be the sum of the excursions of the sarcomeres lying in series; hence, the absolute shortening ( $\mathrm{Sm}$ ) will be equal to the per-sarcomere (relative) shortening (Ss) times the number $(\mathrm{n})$ of serially placed sarcomeres $[\mathrm{Sm}=\mathrm{Ss}(\mathrm{n})]$. Similarly, the absolute velocity of the muscle end $(\mathrm{Vm})$ will be the sum of the per-sarcomere velocities (Vs) of the number of serially placed sarcomeres (cf. Josephson, '75, Fig. 2, drawn for twitch duration). Thus, $\mathrm{Vm}=\mathrm{Vs}_{1}+\mathrm{Vs}_{2}+\mathrm{Vs}_{3}+\ldots \mathrm{Vs}_{\mathrm{n}}$.

If the muscle is pinnate, the principles change. The vectors of the sarcomere strings intersect the line of action of the entire muscle at an angle that changes as the strings shorten. Only the resultants parallel to the line of action contribute useful force. Consequently, a fraction of the force contributed by each sarcomere is lost. However, in bipinnate (and multipinnate) muscle, the shortening of each fiber is amplified so that the tendon moves further than any muscle fiber shortens; this effect reduces the shortening velocity required of each fiber, and hence, increases the force the muscle fiber generates (Fig. 10).

The shortening of an inclined fiber generates an indeterminate movement that may be rectilinear (along the inclined line), rotational (about the origin of the fiber), or a combination of these (Gans, '82). Only if the fibers insert on rigid surfaces or if the surfaces of origin and insertion slide by each other in parallel does one observe the regular changes of pinnation angle with contraction that generally are illustrated in textbooks. However, any increase in the pinnation angle of the muscle fibers (owing to contraction) tends to increase the linear excursion of the entire muscle over that of each component fiber. In other words, the excursion of a bipinnate (or multipinnate) muscle will be greater than the excursions of its component muscle fibers, i.e., $\mathrm{Sm}>\mathrm{Ss}^{\mathrm{n}}$.

Several of these considerations affect the contraction force, because of the way they effect the range of their length/tension curves through which the component sarcomeres act (Fig. 10). Increase of the number of sarcomeres placed in series reduces the excursion required of each, but modifies the absolute length of serially arranged contractile material within a muscle. Whereas the absolute force generated by the individual sarcomeres might remain the same, it may be easier then to match the distance through which each sarcomere shortens to the plateau region of the length-tension curve and, thus, to keep the force output of the sarcomere high (Fig. 10A, B). As noted above, the increase in sarcomere number also reduces the shortening velocity required of each sarcomere. This increases the force output, be- 
cause the general rule is that increased velocity reduces force output, and decreased velocity increases it. Because the relation between load and fiber-contraction rate is nearly hyperbolic, the reduction of velocity will generate an exponential improvement on the force output of each sarcomere.

Pinnation also has two effects, and one of these reflects the changed velocity (Fig. 10C). If the system remains in isometric contraction, the force relations reflect the possibility of packing more fibers into functional parallel. However, if the velocity of shortening

A
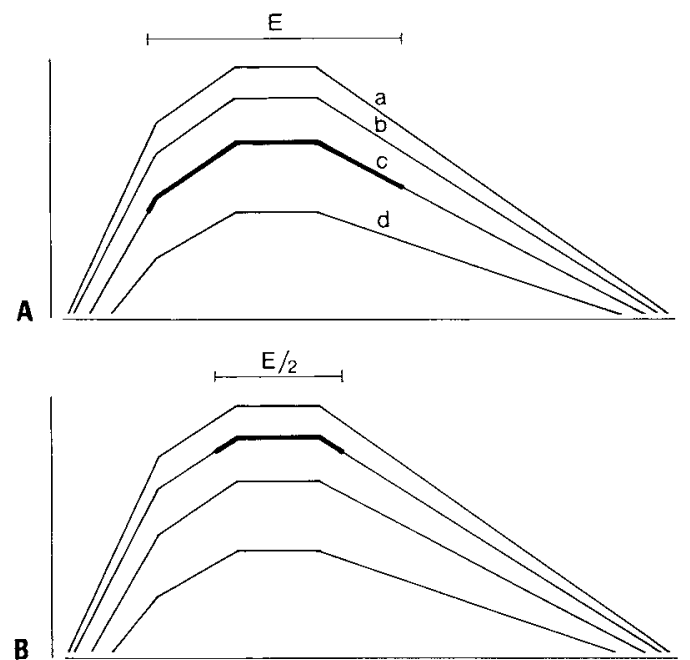

$\frac{5}{\circ}$
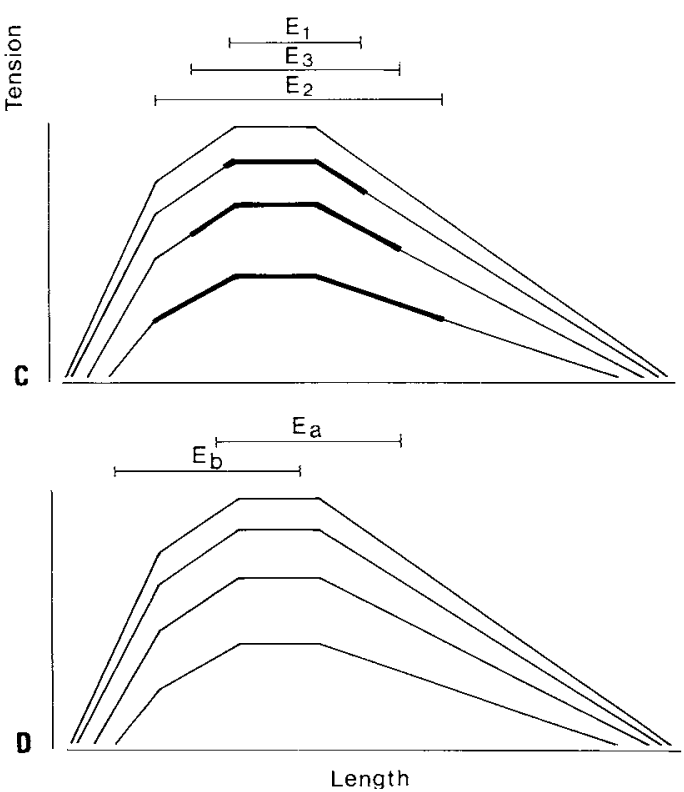

also is considered, the effect is major. As the number of sarcomeres in series is reduced, the movement range of each must increase. This is disadvantageous. However, an increase in excursion of the central tendon also reduces the excursion range required of each sarcomere; the two opposite effects partially compensate so that the potential for maintaining the sarcomere in an advantageous position on the length-tension curve is not affected (cf. Muhl, '82).

If the absolute displacement (velocity, acceleration) of the insertion sites is fixed, the relative shortening of the muscle fibers depends upon their length. For any set of fixed attachment sites, relative shortening will be minimal if the fibers occupy the full distance between origin and insertion of the mass. Relative shortening (and velocity) will increase if part of this distance is occupied by connective tissues rather than sarcomeres.

\section{RULES FOR ANALYSIS \\ Conflict in explanation}

The principles of fiber position and fiber angulation within a muscle generate synergistic, additive, and conflicting effects. Hence, tests need to be designed to establish whether the implications of alternative arrangements

Fig. 10. Length-tension curves of a sarcomere showing its relation to the relative excursion (E) imposed on it. (The relative excursion is the excursion of the end of the muscle divided by the number of sarcomeres in series). The length-tension curves are plotted in terms of Figure 2. In each example, (a) represents isometric contraction and $(\mathrm{b}-\mathrm{d})$ shows increasing degrees of shortening (velocity). A. The excursion overlaps the major portion of the range. The shortening velocity proceeds along curve (c). B. The relative excursion has been reduced (E/2) by increasing (in this case doubling) the number of sarcomeres in series. This maintains the excursion range closer to the maximum force plateau. Reduction of the excursion required of any sarcomere reduces the velocity, so that the force generated is greater, being mapped along curve (b). C. Effect of pinnation. $E_{1}$ is the relative excursion of a parallel-fibered muscle. As it becomes pinnate, the excursion tends to be increased to $E_{2}$, because fewer sarcomeres are in series. However, the theoretical excursion value $E_{2}$ is greater than the actual excursion value $E_{3}$, which benefits from the fact that excursion of the central tendon of a bipinnate muscle is greater than the excursion of the individual fibers. D. Should the maximum demand for force occur near one end of the excursion range, the sarcomere position can be adjusted so that this excursion correlates with the end of the excursion. $E_{a}$, excursion range adjusted so that the force peaks near the shortest excursion. $E_{b}$ excursion range adjusted so that the force peaks near the longest position. The relative shortening is not affected. 
need to be considered in analysis of the observed array. For instance, it remains necessary to check whether the sites at which muscles are placed differ interspecifically and whether the functional (i.e., performance-associated) implications of these differences match possible differences of role. If they do not, it is likely that the character state reflects phylogeny more than function.

The ontogenetic development of muscle is classically flexible. Particularly in mammals, we see multiple levels of physiological adaptation, the pattern of exercise as an obvious determinant. It seems worthy of note that neither epigenetic development $(\mathrm{L} \phi \mathrm{v}$ trup, '75) nor physiological adaptation (Prosser, '58) should be assumed to reflect some intrinsic capacity of cells independent of adaptation. It is more likely that the cellular properties in the past have been selected to provide the organism with another level of matching environmental circumstances (Gans, '66, '74, '79). Hence, there is no reason to treat these processes as an alternative to those of selection.

Also, it is essential to increase the range of taxonomic outgroups selected for comparison and to consider the extent to which the patterns seen reflect structural constraints or developmental factors, although both of these characteristics tend to interact with phylogeny. Epigenetic influences, such as physiological adaptation (i.e., in response to exercise), differ among vertebrates. Furthermore, these are not independent of adaptive influences. Still, one may be able to see whether and why some other structural variants are mechanically unlikely. However, tests of any hypotheses about evolutionary mechanisms demand schemes for estimating the force output and the excursion and velocity patterns of the muscles studied.

\section{Potentially desirable roles}

It has been noted above that it is unlikely that most muscles are selected for a single isolated variable, such as a force or moment arm. Similarly, it is unlikely that a single energetic component, such as the power (i.e., force times velocity) liberated over an entire excursion range, represents the role reflected in all muscle fibers. Indeed, the task proposed here is one of investigating the various potential design parameters and establishing whether and how they are involved in a particular muscle and kind of individual. It seems useful to start by considering some of the characteristics that might be advantageous in different muscles.
The first consideration is the stability of task. Is the muscle (or part of a muscle) under study subject to a single, narrow design criterion to carry out only a single task (with other muscles recruited for other tasks), or must it perform several different tasks? What is the range of excursions across which some action must be generated, and does this range involve a series of static loadings or a continuous shortening (or lengthening) event? What is the rotational (displacement) position at which maximum force is to be exerted? This set of questions may be considered in terms of the distinct force-application regimes that are likely to be encountered, whether in food-processing or in locomotor systems. In the following account, we perform this analysis for a standard sarcomere type involving a particular force-time characteristic.

Certain generalizations pertain to all muscle systems. The mass of muscle incurs costs in its initial formation, in its maintenance (basal and contractile metabolic rates), and because the mass generates inertia whenever the animal moves. The first cost may be reduced by reducing the mass of the muscle, the second by reducing the basal metabolic rate, and the third by locating the muscles in a more proximal position. The cost of muscular effort may be reduced further by matching the demand for output force (over part of the skeletal excursion range) with an optimized combination of the moment arm of each muscle and the length-tension curve of its component sarcomeres. Such matching might be possible because, for example, the insertion angle at the minimal-length position determines whether the rotational moment arm will increase or decrease as the muscle length increases.

The absolute magnitude of the force to be generated by a muscle is the first and major variable, even though this level of force includes compromises for excursion and velocity. The force generated by a sarcomere is, of course, related to the length at which the sarcomere is activated and the relative range and velocity through which excursion proceeds. The force also reflects the compliance, or mechanical permissiveness, of the system, i.e., its tendency to move as forces are applied. If the muscle can be stimulated in a position close to isometric, it will generate a different output than if it is permitted to shorten or if it is stretched at the time of stimulation. Muscle output will depend upon the direction in which the associated joint(s) will permit motion. Such movement will be 
affected by the resultant action of all connected muscles, as well as by the inertia of the system (which is likely moving because of prior action by other muscles).

This suggests that understanding of muscle "design" might start by consideration of 1) the absolute range of movement required by the current insertion, 2) the force required in each portion of this range, and 3 ) the rate at which the force must be generated. The number of sarcomeres placed in parallel (i.e., number of fibrils) will be a key determinant of the force that a muscle can generate. However, amount of force also reflects the excursion level and the range (and velocity) across which the sarcomere will have to shorten (Fig. 10). For instance, different arrangements are required if the muscle must produce its maximal force near its shortest position (e.g., if mandibular adductors act in grinding food with the jaws nearly closed), rather than if the maximal forces occur near the longest position (e.g., with the jaws open in a gape) ('Thexton and Hiiemae, '75; Gans et al., '85).

Design optimization of muscle also should take into account other properties of the system. For instance, the bending movements within the bones forming the lever systems (i.e., those lying parallel to the performance line) obviously are affected by the magnitudes and sites of muscle location (cf. Currey, '84). Greater potential moments must result in systems with heavier skeletal elements and, consequently, greater rotational inertia; however, the mass of the inserted muscles also incurs inertia. More important may be conflicting demands for movement in two opposed directions (i.e., stance-flip phase in locomotion or open-close in mastication). It then may be desirable to consider an adaptive compromise between the muscle locations for these conflicting roles, e.g., the placement of the heavier of two such muscles into the position of least inertia (i.e., closest to the center of rotation of the element).

Several simple principles characterize the relation of sarcomere properties and those of an entire muscle:

1. The isometric force of a muscle is that generated by the number of cross-bridges among the half-sarcomeres lying in functional parallel at any level between muscle origin and insertion. If this number of sarcomeres differs among levels along the length of the muscle, the minimum value pertains.

2 . The excursion of a muscle is equal to the per-sarcomere excursion multiplied by the number of sarcomeres in series between ori- gin and insertion. If the number of sarcomeres along this distance differs, so will the per-sarcomere excursion.

3 . The velocity attained by the end of a muscle will be the per-sarcomere velocity multiplied by the number of sarcomeres in series between origin and insertion.

4. The greater the per-sarcomere velocity, the less the per sarcomere force produced for any given starting position. Similarly, an increase in the per-sarcomere excursion likely decreases the per-sarcomere force; however, this relation depends upon the portion of the length-tension curve over which shortening takes place. The addition of such forces follows principle 1 .

The behavior of a muscle, then, reflects the environment of its location. Once stimulated, a muscle, for example, may act to accelerate its insertion site. The compliance of this site (i.e., the force required to accelerate it to a particular velocity) determines the rate at which the insertion site can be moved. However, as the potential velocity of the insertion site is increased, the force each sarcomere of the muscle can produce falls. (Theoretically, a muscle could contract more slowly than its site of insertion is moved by other factors; this muscle, then, would not contribute to the movement.) The intersection of the force-velocity relations, respectively, of the muscle and of the linkage, establishes the particular force and velocity apt to be achieved. These characteristics establish the parameters that describe how the system can be modified in particular organisms. In tabulating these, we introduce some redundancy, because it is obvious that we cannot improve all aspects simultaneously.

Other things being equal, increased force can be attained from a given mass of muscle by 1) placing more of its sarcomeres into physiological parallel (perhaps by pinnation), 2) reducing the per-sarcomere excursion, thereby maintaining all parallel sarcomeres near the peak of their length-tension curve, and 3) reducing the per-sarcomere velocity of shortening. (Factors 1 and 3 will be in conflict.) Force also can be increased by increase of the muscular mass.

Absolute excursion against a particular force can be increased for a given mass of muscle by 1) placing more sarcomeres into series (perhaps lengthening the entire muscle by increasing the distance between origin and insertion of the contractile zone) and 2) reducing the per-sarcomere velocity of shortening so that these may contract to a position further down the left-hand slope of the 
length-tension curve, an effect also achievable by (1). Increase of the physiological crosssection of the entire muscle (by increasing its mass) also imposes less of a force demand on each sarcomere and permits it to shorten further.

Increased velocity can be attained by 1) placing more sarcomeres into physiological parallel, thus reducing the force demand upon each of them, and 2) concentrating the activity of each sarcomere near the peak of its length-tension curve (which may require placement of more sarcomeres in series). The increased velocity may be limited because the velocity of the stimulus along the fiber may be less than the velocity of shortening of the fibers themselves; however, in long arrays this can be compensated by placing multiple, independently innervated fibers end to end (Loeb et al., '87).

In our example, the length-tension relations of the jaw musculature are affected directly by the selected gape, which determines the amount of excursion required of the mandible and the muscle. Geometric modifications might increase linear gape as measured at the tips of the incisors. These modifications are: lengthening of the jaw anterior of the insertion, shifting the insertion site of the muscle, and shifting the site of origin of the closing muscles (perhaps by use of tendinous pulleys, which also permits more sarcomeres to be placed into series). Horizontal movements of the mandibles might require shallow insertions of the adductor muscles.

The requirement of a force pattern during closure of the jaws is affected by multiple behavioral components. Carnivores might achieve selective advantage by rapid opening and even more rapid closure in order to trap prey. In herbivores, jaw opening might be matched to the need to reposition food during the masticatory cycle. Hence, the initial (although "fast") closing may be slower than in carnivores. Both during fast-opening and fast-closing, the effect must reflect mainly the force required to move the mandibular mass; thus, the initial accelerational force may be limited. Moreover, during such initial acceleration, the sarcomeres of the adductors will be in the most extended portion of their length-tension curve. Slight temporal overlap with the activity of the opening muscles might amplify the closing force by activating the adductor muscles in extension.

The force required increases sharply as the jaws impact upon and deform the food item (hence "slow" closing). In mammals and a few other forms, after impact, the jaws swing horizontally marking the onset of a low excursion-high force (grinding-crushing) phase. In some species, the action is subdivided between two sets of muscles, with one set (that maintaining closure) operating nearly isometrically.

For the grinding-crushing interval, selection may be for increased power generated by the muscle. It has been proposed that force times velocity over the entire excursion range can be maximized in such systems (Currey, '84). There is also the possibility that the momentum of the moving head, jaws, and soft tissues might store kinetic energy that subsequently is expended on the reduction of certain foods (cf. Kallen and Gans, '71). It would be interesting to check whether the mass of the rotating portions correlates with masticatory frequency.

These patterns suggest that masticatory forces generally are applied while the mouth is almost closed, the adductors are near their shortest position, and propalineal movements are often seen. However, the preceding analysis emphasizes the process of mastication more than that of initial food acquisition. Both the killing bites of carnivores and the kinds of reduction bites (for instance, segmenting fruit and tubers or peeling branches) may require force application at greater gapes. Also, we do not know enough about the influence of occasional peak forces, for instance those involved in the crushing of seeds and shells (Janzen and Martin, '82; Irish, '83; Gans and de Vree, '86). Moreover, both carnivores and herbivores might use their teeth in other activities such as fighting (e.g., male horses or hippopotami), subduing females (e.g., lizards and sphenodontids), and digging (e.g., mole rats). Hence, the advantage of applying particular force levels at various degrees of closure likely differs among species. Without measurement, it cannot be assumed to be constant.

\section{Parameters to be established for quantification of muscular pattern}

Calculation of the forces and displacement generated by muscles requires several levels of information about the nature and overall architecture of the muscles involved. Although the preceding discussion has alluded to these, they seem important enough to be listed specifically.

An outline for the study of quantification of muscular patterns can be discussed in the following six overlapping stages: 
1. Establish the excursion pattern (displacement and rate) of the system by observation and film analysis and correlate translational and rotational movements with fiber (sarcomere) length. Establish the time course of movement and force, i.e., is force applied to prey slowly or rapidly?

This initial step requires characterization of the excursion pattern (kinematics and dynamics) of the movable elements. The main questions are: what are the degrees of freedom at the several joints? What is the stiffness of the component elements, indeed the compliance of each joint as a function of the degree of rotation or translation? Will there be elastic deformation of the skeletal parts?

2. Establish the architectural pattern of muscle arrangements. Consider the geometry at the limiting conditions (open-close) and note whether the intermediate positions are linear.

Detailed information is required about the attachment pattern of the muscle masses and the way that positions of muscles change as the system shifts through its range of motion. Do the fibers attach to tendons or aponeuroses that in turn attach to skeletal elements, or are the fibers connected directly to a bony surface in a fleshy pattern? Is the insertion site of the muscle loose so that the entire muscle is likely to shift to a straightline position between the insertion and the origin, or are the individual fibers and their surfaces of attachment constrained to a particular position?

Another set of questions considers details of fiber length and attachment. How long are the fibers? How are the fibers angled? What is the variability of these factors? The angles of the fibers should be established relative to a) the long axis of the muscle itself, b) the site of fiber insertion (in terms of length along the moment arm and height above the performance line), and c) the performance line. Also, the change in muscle length and angle with movement should be noted. This must occur at least at the terminal (open/close, protrude/retract) or functionally most important (grasp, crush) positions; better yet, it should be recorded throughout the entire range of motion. Positions and angles are occupied and formed in three-dimensional space; hence, these parameters must be checked independently along the multiple possible axes of organization.

The most important component, here and in the next section, is the determination of functional subdivisions of the muscle. Rather than relying on the muscle names, which tend to be constrained by search for homology, it becomes necessary to identify those groups of fibers that differ from adjacent ones by any characteristic that is likely to affect the force they exert and the time and joint position at which they exert it.

3. Determine the general fiber type of the muscles. Response not only to twitch, but also to tetanic activation, should be considered. This information indicates the time course of force generation (and the excitation-contraction coupling so important in the interpretation of electromyograms). Information about the relative fractions of the (physiological) cross-sectional area for each fiber type also is needed. These values, used in combination with the data generated from the other stages, will characterize the magnitude, direction, and time course of the vectors likely to be produced by each fiber grouping. (Include the same information for each of the several motor units if these can be defined.)

It is also important to note the actual length of the fibers, rather than that of the fascicles comprising them. Various packing systems have been described in which short fibers lie end to end or show slight overlap. In other arrays, such as the axial musculature of some snakes, short segments of muscle attach to longer segments of connective tissues. The compliance of such attachments must be documented. Also, it is uncertain how much of the force generated in such systems is transmitted via tendons and how much is passed laterally to the fibers of adjacent segments.

4. Establish the length-tension curve for each muscle. Generate curves for different velocities and for lengthening and shortening (use information from the next stage). The effects of pinnation can be included here or considered at a later stage. In the former case, establish the curve for the entire muscle, rather than for each set of fibers or motor units.

Naturally all of the preceding section must be considered relative to the fiber types involved and the motor-unit territories. Action of a muscle must be evaluated in terms of the activity of each portion through time, particularly if the anatomical muscle contains multiple populations of motor units with distinct roles.

5. Use electromyographic data to establish the time (and length) during movement at which each muscle group is active. If possible, establish which portion (fiber groupings, motor units) of the muscle is used. Also, check 
whether the activation pattern is regular (isolated spikes), or whether there exists the potential for the occurrence of temporal summation (tetanus).

6. Calculate the dynamic pattern of force application for each instant during the movement cycle of the mechanical system under study. Attempt to measure the individual forces or the force generated by the entire set that is activated. Determine how the dynamic pattern matches the presumptive roles of the system.

This outline for quantification indicates that an architectural analysis only provides the first stage of this analytical process. However, it confirms that architectural analysis is fundamental to the remaining steps.

Assessment of intraspecific variability remains critical throughout. It must be taken into account in determining fiber length and angle (but also for the other levels of comparison). Extreme morphological variability might indicate that the muscle is compound, subserving several roles. Extreme physiolog. ical variability might indicate that the parameter being quantified may be a biologically coincidental byproduct, i.e., that we are looking at a component different from the one on which selection acts. In contrast, limited variability might suggest that the parameter is important to the organism (Gans and Gorniak, '82; Shaffer and Lauder, '85).

Given these steps toward quantification, one should be able to determine the relative force magnitude of any component muscle and, thus, circumscribe the potential significance of the muscle. Quantification also allows estimation of the effects of potential manipulation on the system. For instance, the cost of flattening or arching a vertebrate skull can be estimated, not only in terms of modified gape, but also in terms of the changed distance between (and need for modification of) origin and insertion of the muscles. Such models have the practical power of permitting one to estimate the effect of muscle replacement by surgery.

\section{Calculation of force from muscle locations}

It is preferable to measure forces directly; however, often this is impractical. The following principles provide an interim solution applicable in comparative studies, for example. The contraction of a fiber applies force to both ends of fiber attachment. The vector of the force will be approximately in line with the position of the stimulated fiber. Consequently, the contraction of a set of equivalent fibers positioned parallel to each other should generate a force that can be envisioned to act at the geometric center of the surface of attachment (origin or insertion).

This generalization permits a simple solution for the force transmitted by parallelfibered muscles, the fibers of which have equivalent length and attach to a compliant tendon that permits rotation of the entire muscle about the attachment site as the joint undergoes rotational motion. There remains the caveat that the muscles are subdivided into functional groups, and only a fraction of their component mass of fibers is likely to be active for any one task. For any relative shortening (velocity), at which the mean force (of the activated fibers) may be estimated as "f"' (stage 3 , above) in Newtons $/ \mathrm{cm}^{2}$, the relation for the force $F$ generated by the entire muscle will be $F=f \cdot V L$, for which $V$ is the volume of the muscle (in $\mathrm{ml}$ ) and $\mathrm{L}$ the length of its component fibers (in $\mathrm{cm}$ ). If this calculation is to proceed on the basis of muscle mass $M$, then $V=M / d$, where $d$ is the density of the muscle in equivalent units.

If the muscle has pinnately arranged fibers of constant length and is free to rotate at its ends so that it generates translational force, the force generated by the entire muscle will be $F=f \cdot \cos a \cdot V L$, for which $a$ is the mean angle at which the muscle fibers are activated. This mean angle must be determined relative to the aponeurosis of insertion, rather than to any of the major axes of the body. (Note again that the trigonometric term may be omitted if the fibers of the pertinent portions of the muscle are restrained and only permitted to shorten in the pinnate position during the determination of the value $f$. Their force vectors then are generated at the same angle as in the intact animal.)

A more complex situation arises whenever the variously angled fibers are attached to bony extensions or to aponeuroses in a rotating system that places their sarcomeres into functional equivalence (see above). Assuming that the aponeuroses are not permitted to comply and that the fibers act equivalently (i.e., as if they inserted on the performance line at identical angles), the relation for the force $\mathbf{F}$ at the tendon of such a muscle will be $F=f \cdot V L_{\text {mean }}$, for which $L_{\text {mean }}$ is the mean fiber length for the entire muscle. Unfortunately, it cannot be assumed that the force of such a muscle acts at the geometric 
center of the attachment area. Insertion of the fibers off the performance line by attachment to bony and aponeurotic surfaces increases the complexity of the arrangement and the range of individual vectors. However, it is possible to approximate the force vectors by estimating the mean length for the set of muscle fibers. It is then appropriate to assume that the center of action will correspond to the position (along the performance line) occupied by fibers of equivalent length. A similar correction must be applied for the vertical displacement off the performance line, which position will also establish the relative angle of inclination of the muscle. Unfortunately, this rule does not establish the position of the resultant in the transverse direction.

A further complication occurs if fibers of unequal length, arranged for functional equivalence of their sarcomere strings, also are pinnate. If the pinnation is regular, the relation for the force $F$ will be $F=f \cdot \cos a \cdot$ $\mathrm{V} / \mathrm{L}_{\text {mean }}$, where a refers only to the (presumbly transverse) angle of pinnation and not to the fiber angulation that generates equivalence. However, if the pinnation is irregular (as when the fibers insert on the proximal end of an aponeurosis at angles other than those reflecting the principle of functional equivalence), the situation becomes indeterminate. Fortunately, most such systems can be estimated by the same relationship with the assumption that the percentage of irregular fibers is insignificant. It is, of course, always important to show whether the "irregularly placed" fibers are really insignificant variants and to test whether their activity is in synchrony with the remainder of the fiber mass. It remains possible that the irregular fibers might contribute to the performance of an unexpected task.

Much of the preceding discussion makes the assumption that sarcomere equivalence is a general phenomenon, i.e., that all of the simultaneously active sarcomeres of each muscle show an equivalent shortening. The tests provided thus far remain to be confirmed both for portions of complex muscles and, perhaps, for some entire muscles. Thus far, estimation of the reality of this assumption has proved difficult because postmortem changes are likely, even in perfused specimens. The reality of local differences in shortening is questionable. Also, there is the corresponding concern that such differences affect the time course of force generation and shortening. We stress the problem because it is likely that newer techniques, such as laser diffraction, soon will permit us to establish the magnitude of differences in relative shortening among sarcomeres, at least of those sarcomeres that comprise the fibers near the surface of a muscle.

\section{CONCLUSIONS}

These simple relationships among sarcomere characteristics provide an initial estimation and comparison of force generation and shortening by muscles with various kinds of architectures. However, the relationships also document that the several kinds of fiber angulation lead to different predictions about the course of force generation and shortening of any system. The pattern often referred to as pinnation describes only a part of the angulations seen in architectural arrays.

Finally, we must note again that the moment arm produced by the forces generated by bridging muscles will change as the positions of the joints change. These factors must be determined for the time and joint positions during which the studied portions of the muscle are active. The forces produced by muscles are needed by the animal (and hence of interest to us) because they generate moments and induce velocities along a performance line. It is the totality, rather than any isolated aspect, of muscle architecture that is likely to be under the influence of selection.

\section{ACKNOWLEDGMENTS}

We thank D. Carrier, A.S. Gaunt, Marc Offermans, and S.-E. Widmalm, but particularly Linda Trueb, for comments. C.M. Bull, A.E. Greer, and G. Grigg helped substantially by a gift of the experimental animals and aid in obtaining export permits. Mr. A. Cuylits kindly prepared the drawings. The initial draft of this manuscript was prepared while C.G. was adjunct professor at the $\mathrm{Mu}$ seum National d'Histoire Naturelle in Paris, and he wishes to acknowledge its hospitality as well as discussions with colleagues there. It is a pleasure to acknowledge that this is one of a number of studies that was pursued while C.G. held a guest professorship at the Department of Biology of the University Antwerp (UIA). This work was supported by NSF grant G-BSR-8509490. 


\section{LITERATURE CITED}

Akster, H.A. (1984) Muscle Fibre Types of Fishes. Struc tural and Functional Specialization. Ph.D. dissertation, Univ. Wageningen.

Burke, R.E. (1978) Motor units: Anatomy, physiology and functional organization. In The Handbook of Physiology: The Nervous System II. Bethesda, Maryland: U.S. Department of Health and Human Services, pp. $345-422$.

Currey, J. (1984) The Mechanical Adaptations of Bones. Princeton, New Jersey: Princeton University Press.

Focant, B., H. Benmouna, and P. Vandewalle (1985) Comparison of the myosin and parvalbumins of head and trunk muscles from Serranus scriba (L.) and Serranus cabrilla (L.). Arch. Int. Physiol. Biochim. 93:B82.

Focant, B., and A. Pequeux (1985) Comparative electrophoretic study of myosins and parvalbumins from three deep sea fishes. Mora mora R., Conocara murrayi K. and Coryphaenoides rupestris G. Bull. Soc. Roy. Sci. Liege 54(1):55-64.

Gans, C. (1966) Some limitations and approaches to problems in functional anatomy. Folia Biotheor. 6:41-50.

Gans, C. (1974) Biomechanics. An Approach to Vertebrate Biology. Ann Arbor: The University of Michigan Press.

Gans, C. (1979) Momentarily excessive construction as the basis for protoadaptation. Evolution 33:227-233.

Gans, C. (1982) Fiber architecture and muscle function. Exerc. Sport Sci. Rev. 10:160-207.

Gans, C., and W.J. Bock (1965) The functional significance of muscle architecture: A theoretical analysis. Adv. Anat. Embryol. Cell Biol. 38:115-142.

Gans, C., and G.C. Gorniak (1982) Protrusion of the tongue in marine toads (Bufo marinus). Am. J. Anat. 163:195-222.

Gans, C., F. de Vree, and D. Carrier (1985) Usage pattern of the complex masticatory muscles in the shingle-back lizard, Trachydosaurus rugosus: A model for muscle placement. Am. J. Anat. 173:219-240.

Gans, C., and F. de Vree (1986) Shingle-back lizards crush snail shells using temporal summation (tetanus) to increase the force of the adductor muscles. Experientia 42:387-389.

Gaunt, A.S., and C. Gans (1969) Mechanics of respiration in the snapping turtle, Chelydra serpentina (Linné). J. Morphol. 128: 195-228.

Gordon, A.M., A.F. Huxley, and F. Julian (1966) The variation in isometric tension with sarcomere length in vertebrate muscle fibers. J. Physiol. 184:170-190.

Gorniak, G.C., H. Rosenberg, and C. Gans (1982) Motor activity during mastication in the tuatara, Sphenodon punctatus (Reptilia: Rhynchocephalia). J. Morphol. 171:321-353.

Guthe, K.F. (1981) Reptilian muscle: Fine structure and physiological parameters. In C. Gans and T.S. Parsons (eds): Biology of the Reptilia, Vol. 11. London: Academic Press, pp. 265-354.

Herring, S.W., A.F. Grimm, and B.R. Grimm (1984) Regulation of sarcomere number in skeletal muscle. A comparison of hypotheses. Muscle Nerve 7:161-173.

Hoyle, G. (1983) Muscles and Their Neural Control. New York: John Wiley \& Sons.

Huddart, H. (1975) The Comparative Structure and Function of Muscle. Oxford: Pergamon Press.

Irish, F. (1983) The dynamics of seed-eating in the characiform fish Colossoma. Am. Zool . 23:1027.

Janzen, D., and P.S. Martin (1982) Neotropical anachronisms: The fruits the gomphotheres ate. Science 215:1927.

Josephson, R.K. (1975) Extensive and intensive factors determining the performance of striated muscle. J. Exp. Zool. 194:135-154.
Kallen, F.C., and C. Gans. (1971) Mastication in the little brown bat, Myotis lucifugus. J. Morphol. 136:385-420.

Levine, R.J.C., M.M. Dewey, and G.W. Villafranca (1972) Immunohistological localisation of contractile proteins in Limulus striated muscle. J. Cell Biol. 55:221-235.

Loeb, G.E., and C. Gans (1986) Electromyography for Experimentalists. Chicago: University of Chicago Press.

Loeb, G.E., C.A. Pratt, C.M. Chanaud, and F.J.R. Richmond (1987) Distribution and innervation of short, interdigitated muscle fibers in parallel-fibered muscles of the cat hindlimb. J. Morphol. 191:1-15.

Løvtrup, S. (1975) Epigenetics. New York: John Wiley \& Sons.

Mashima, H. (1984) Force-velocity relation and contractility in striated muscles. Jpn. J. Physiol. 34:1-17.

McMahon, T.A. (1984) Muscles, Reflexes and Locomotion. Princeton, New Jersey: Princeton University Press.

Muhl, Z.F. (1982) Active length-tension relation and the effect of muscle pinnation on fiber lengthening. J. Morphol. 173:285-292.

Otten, E. (1987) Optimal design of vertebrate and insect sarcomeres. J. Morphol. 191:49-62.

Prosser, C.L. (ed.) (1958) Physiological Adaptation. Washington, D.C.: American Physiological Society.

Shaffer, B., and G. Lauder (1985) Aquatic prey capture in ambystomatid salamanders: Patterns of variation in muscle activity. J. Morphol, 183:273-284.

Stern, J.T., Jr. (1974) Computer modelling of gross muscle dynamics. J. Biomech. 7:411-428.

Thexton, A.J., and K.M. Hiiemae (1975) The twitch-contraction characteristics of opossum jaw musculature. Arch. Oral Biol. 20:743-748.

Vandewalle, P., T. Monfils, F. Huriaux, and B. Focant (1983) Activités musculaires des faisceaux rouges et roses de l'adducteur de la mandibule chez la carpe (Cyprinus carpio L.) pendant la respiration et la toux. Ann. Soc. Roy. Zool. Belge 113:107-114.

Weijs, W.A., and T.K. van der Wielen-Drent (1982) Sarcomere length and EMG activity in some jaw muscles of the rabbit. Acta Anat. 113:178-188.

Weijs, W.A., and T.K. van der Wielen-Drent (1983) The relationship between sarcomere length and activation pattern in the rabbit masseter muscle. Arch. Oral Biol. 28:307-315

Wineski, L.E. and C. Gans (1984) Morphological basis of the feeding mechanics in the shingle-back lizard Trachydosaurus rugosus (Scincidae: Reptilia). J. Morphol. 181:271-295.

\section{APPENDIX}

Tables 1 and 2 present the results of measurements of fiber length and placement in the very complex $M$. adductor medialis externus, part 2 (MAME-II) of the skink, Trachydosaurus rugosus (see Wineski and Gans, 1984 , for anatomical terminology). The origin and insertion of each fiber relative to the position of the midsagittal plane and the performance line was determined by means of a stereotactic apparatus (Table 1). Seventy fibers were measured on a single specimen freshly preserved for this purpose and the values compared with those for three other heads of specimens sacrificed after experiments. Repeatability of measurement was established by removing and replacing the 


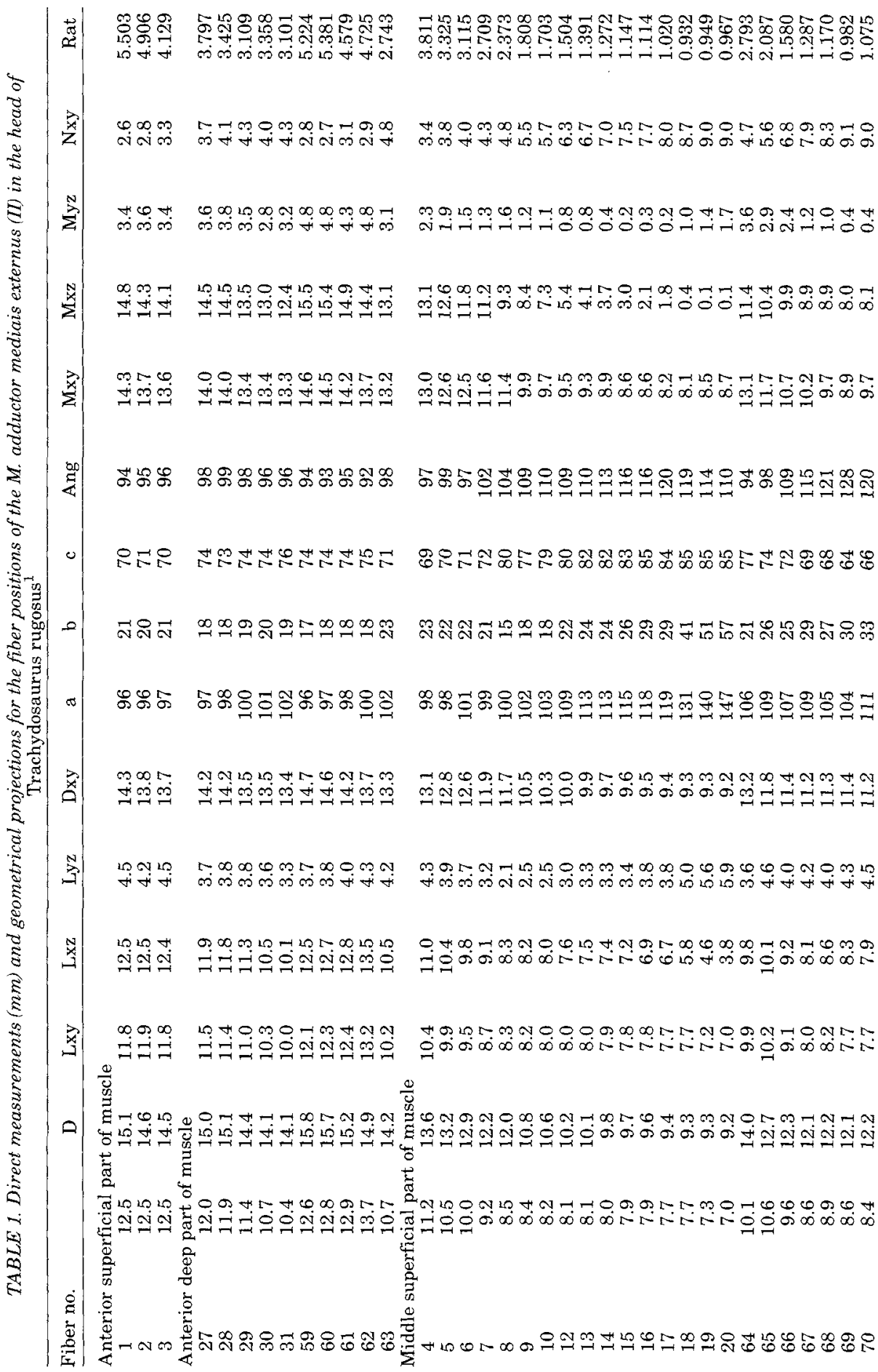




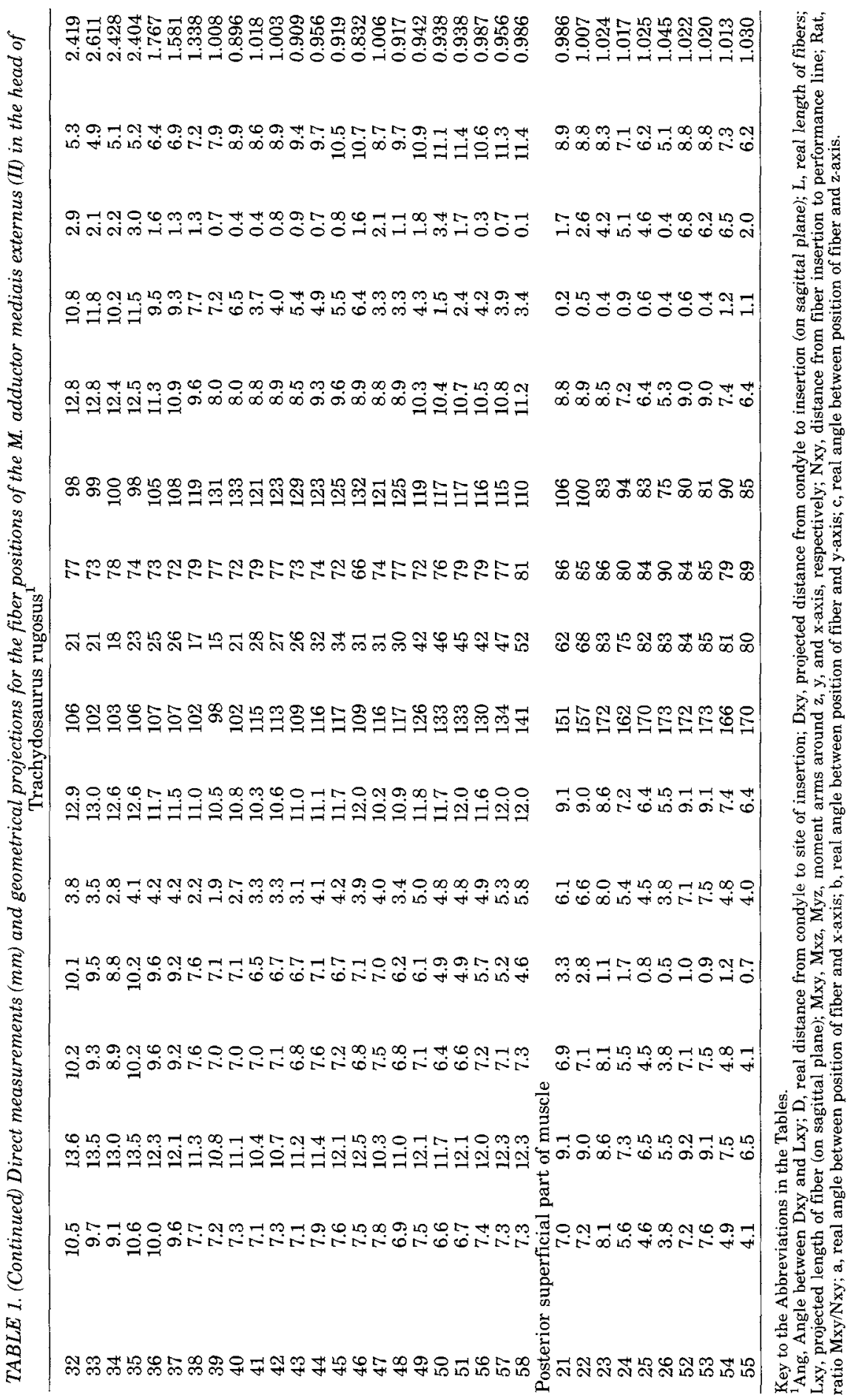




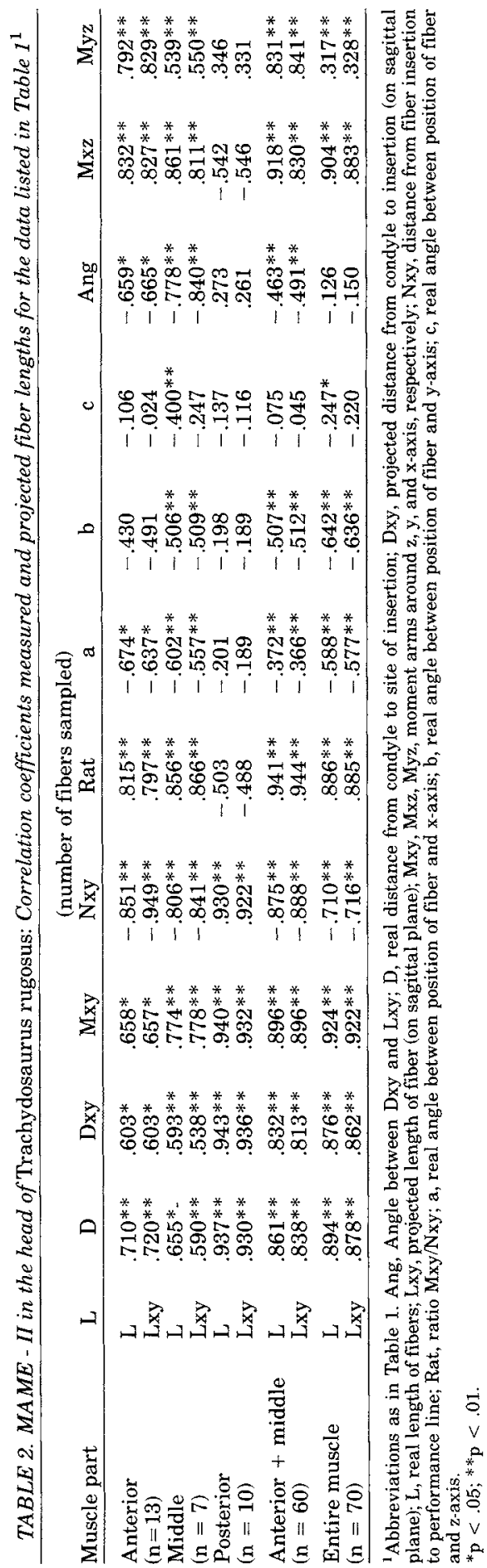

head from the stereotactic apparatus and remeasuring the position of marked fibers. Angles relative to the midsagittal plane were determined and the absolute fiber length was calculated from the rules of solid geometry and resolved into values on the midsagital plane (that of rotation and here of equivalence). Absolute angles $a$ and $b$ (respectively, between the fiber and the $\mathrm{x}$-axis and $\mathrm{y}$-axis) corresponded to this value. Absolute angle $c$ (between the fiber and the $\mathrm{z}$-axis) reflected the pinnation of the muscle, although it was not the real pinnation angle, as this has to be resolved in the plane in which the fiber lies. The muscle was subdivided into portions, which reflect the spatial constraints forced, for instance, by the passage of the MAME-I.

Table 2 correlates the values for each measurement against absolute and projected muscle length. In general, the values as summed for the entire muscle are highly significant. As fiber length correlates significantly with such measures as the distance from insertion to the condyle, as well as to the moment arm around the axis of mandibular rotation, the analysis agrees with an earlier, less detailed one (Gans et al., 1985; based upon three specimens) and supports the concept that sarcomere equivalence occurs throughout the muscle. The differences in correlation for parts of the muscle apparently reflect sample size. The low correlation between muscle length and angle c presumably reflects the fact that this angle only reflects pinnation for fibers that lie in the transverse plane (i.e., that have an angle of $90^{\circ}$ with the performance line). Furthermore, the muscle in question shows minimal pinnation, as defined here. 